\title{
Modeling and Control of TCV
}

\author{
Atul S. Sharma, David J. N. Limebeer, Fellow, IEEE, Imad M. Jaimoukha, and Jonathan B. Lister
}

\begin{abstract}
A new approach to the modeling and control of tokamak fusion reactors is presented. A nonlinear model is derived using the classical arguments of Hamiltonian mechanics and a low-order linear model is derived from it. The modeling process used here addresses flux and energy conservation issues explicitly and self-consistently. The model is of particular value, because it shows the relationship between the initial modeling assumptions and the resulting predictions. The mechanisms behind the creation of uncontrollable modes in tokamak models are discussed. A normalized coprime factorization $\mathcal{H}_{\infty}$ controller is developed for the the Tokamak à Configuration Variable (TCV), CRPP-EPFL, Lausanne, Switzerland, tokamak using the linearized model, which has been extensively verified on the TCV and JT-60U, JAERI, Naka, Japan, tokamaks. Recent theory is applied to reduce the controller order significantly whilst guaranteeing a priori bounds on the robust stability and performance. The controller is shown to track successfully reference signals that dictate the plasma's shape, position and current. The tests used to verify this were carried out on linear and nonlinear models.
\end{abstract}

Index Terms-Control systems, $\mathcal{H}_{\infty}$ control, model reduction.

\section{INTRODUCTION}

$\mathbf{T}$ HE TOKAMAK was conceived by the Russian scientists Tamm and Sakharov, winners of the Nobel Physics and Peace Prizes, respectively. A Tokamak ${ }^{1}$ is a toroidal device (see Fig. 1) that uses magnetic fields to confine a similarly shaped, hot plasma (typically up to $10^{8} \mathrm{~K}$ ). This method of confinement exploits the fact that plasmas are made up of free electrons and ions. As a result, the plasma can be confined using electromagnetic forces generated by external fields. This external magnetic field has two components. The large toroidal field is produced by a set of poloidally wound coils equally spaced around the vacuum vessel and the smaller poloidal field comes from the induced plasma current. The resultant field is helical, as shown schematically in Fig. 1.

As a source of power, nuclear fusion has a number of attractive features. The fuels are abundant, there are no long-lived radioactive isotopes produced and it is inherently safe. Nuclear fusion does not contribute to the emission of gases causing the greenhouse effect, or acid rain. At the time of writing, the tokamak is the most promising route to a viable fusion reactor.

Manuscript received September 23, 2003. Manuscript received in final form August 10, 2004. Recommended by Associate Editor C. Knospe.

A. S. Sharma, D. J. N. Limebeer, and I. M. Jaimoukha are with the Department of Electrical and Electronic Engineering, Imperial College of Science, Technology and Medicine, London SW7 2BT, U.K. (e-mail: d.limebeer@ imperial.ac.uk).

J. B. Lister is with the Centre de Recherches en Physique des Plasmas, École Polytechnique Fédérale de Lausanne, Association EURATOM-Suisse, 1015 Lausanne, Switzerland.

Digital Object Identifier 10.1109/TCST.2004.841647

${ }^{1}$ From the Russian "toroidalnaya kamera magnitnaya katushka," literally "toroidal chamber, magnetic coils."
The basic characteristics of tokamak equilibria, as described by the Grad-Shafranov equation, are well understood [1] and the theory is relatively accessible [2]. In order to model the gross dynamic behavior, it is possible to make a number of approximations that are accurate enough to provide a basis for robust controller design. A number of linearized models suitable for control system design have already been developed. In the case of the CREATE-L, we refer the reader to [3]. Comparable studies have been performed on DIII-D2 [4], [5], and on TCV [6], [7].

Our modeling research is motivated by the facts that improved models will both further our physical understanding and lead to control systems that extend the operating regimes of existing and future tokamaks such as ITER ${ }^{3}$ [8].

We present a lumped-parameter model that is derived using the established processes of classical Hamiltonian mechanics. For the purposes of model-based controller design, this model can be linearized about any prescribed equilibrium state. As compared with the current art, this model has several distinct features. To begin, flux and energy conservation issues are treated explicitly and self-consistently. In [3], for instance, plasma profile parameters are considered as disturbances, which the authors acknowledge is not self-consistent. Previous derivations of the RZIP-type models [so-called as they model plasma radial and vertical position (R, Z) and current (IP)] [6] have also not considered conservation laws in a self-consistent manner. The consistency issue was first addressed in our previous work [9], where a Lagrangian approach to tokamak modeling was introduced. The derivation presented here results in a linear model similar to [6] and [9]. This generalized class of lumped-parameter models have been extensively validated against the open-loop response of TCV [6] and the larger, hotter JT-60U [9]. An earlier, much simpler lumped-parameter model describing only the vertical motion of the plasma using an eigenmode description of the passive structures, was validated on DIII-D [10]. Evidence for shortcomings of the RZIP approach exist only for very triangulated plasmas [7], in which case the deformability of the plasma may play a role. However, in the case of the linearized model, a parametric modeling approach can overcome this limitation [9].

The modeling theory we present here is of particular value, because it shows clearly the relationship between the initial assumptions and the final model. For instance, it becomes obvious that the adiabatic approximation is a natural result of assuming a massless plasma. The next generation of tokamaks will operate on longer time-scales, where plasma resistance effects will become important. Following from [6] and [9], the model presented in this paper considers fully the effects of plasma resistance, showing how to incorporate resistance and mass into the

\footnotetext{
${ }^{2}$ The General Atomics National Fusion Facility, San Diego, CA.

${ }^{3}$ International thermonuclear experimental reactor.
} 


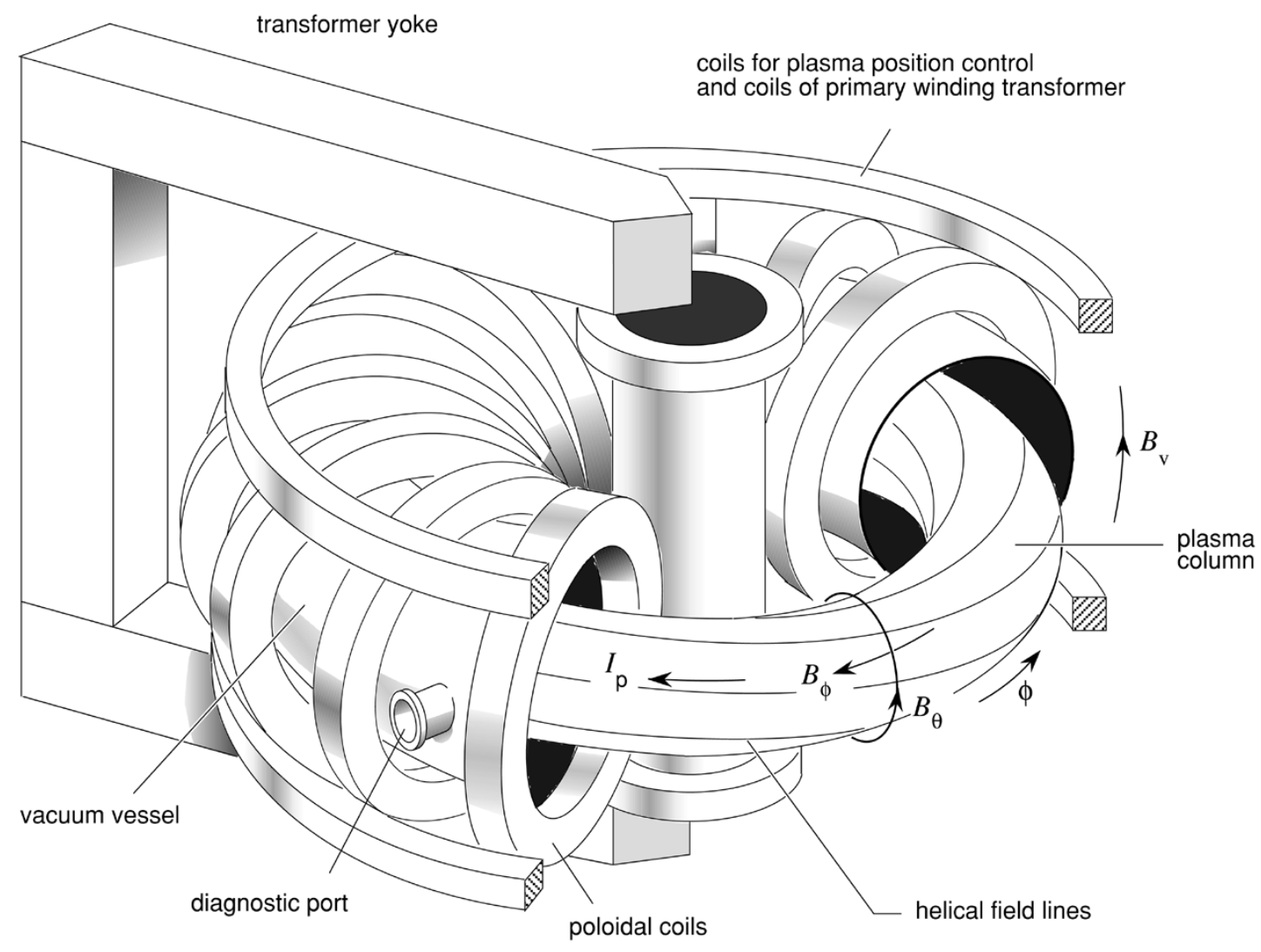

Fig. 1. Diagram of a typical tokamak. Note that the TCV tokamak does not have an iron core (transformer yoke), and has PF 18 coils for position and shape control.

model from the initial stages, if required. The known necessity of current ramping is clearly explained in terms of plasma resistance, as is the creation of uncontrollable modes when a superconducting plasma is assumed. Also, it becomes clear why the use of flux coordinates becomes problematic when plasma resistance is introduced.

A mixed PID- $\mathcal{H}_{\infty}$ controller has already been designed for COMPASS-D [11] and this methodology was developed and then successfully implemented on TCV [12]. The control scheme was based on the CREATE-L model presented in [3]. In [13], a multivariable normalized coprime factor $\mathcal{H}_{\infty}$ controller is designed using a simple single-filament model of the DIII-D tokamak.

High-order $\mathcal{H}_{\infty}$ controllers can present their own implementation problems. The usual approach is to design the $\mathcal{H}_{\infty}$ controller about a reduced order model. In this paper, we develop a reduced order normalized coprime factor controller for TCV that exploits results presented in [14]. We show that this controller extends the performance of the existing PID schemes [7], [15]. This approach guarantees both the stability of the closedloop (via an a priori condition) and the closed-loop performance via an a priori performance bound [14].

In this paper, we apply the theory in [14] to the full $\mathcal{H}_{\infty}$ controller developed for TCV, reducing the controller order from 76 to 18 without a significant loss of performance or robustness. Successful closed-loop tests are performed with the PROTEUS nonlinear tokamak simulation code. The remaining verification step will involve hardware tests on the TCV machine itself.

\section{HAMiLTONiAn TOKAMAK MODEL}

The aim of this section is to develop a dynamic model of the tokamak using established arguments from Hamiltonian mechanics [16]. The development builds on from that given in [9]. The main components of the model are the poloidal field coils, the passive structure, which carries eddy currents, and the plasma. The poloidal field coils are driven by external voltage sources, while the eddy currents in the passive structure are electromagnetically induced. A cylindrical coordinate system is used, with $R$ the radial coordinate, $z$ the vertical coordinate, and $\phi$ the angular coordinate describing rotation around the $z$ axis (see Fig. 2). When deriving the model, we will make the following assumptions:

- the system is symmetric around the $z$ axis (axisymmetry);

- any poloidal currents are ignored;

- the tokamak structure will be represented by a finite set of toroidal circuits.

We also assume that:

- these circuits are fixed in space and have finite resistance;

- the toroidal currents in the structure may vary in time.

- The plasma is represented by a finite number of axisymmetric current carrying elements that have a circular cross section (see Fig. 2). The $k$ th current carrying plsma current element is shown in Fig. 3, in which $R_{k}$ is the the element's radius, $i_{k}$ its current and $a_{k}$ its radius. 


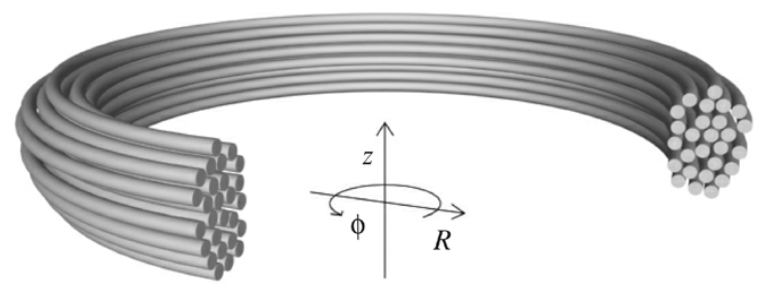

Fig. 2. Plasma is represented by current-carrying elements. The cylindrical coordinate system $(R, z, \phi)$ is shown.

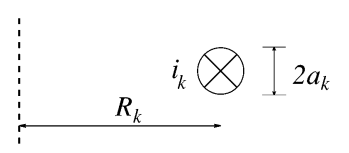

Fig. 3. Plasma current element.

In addition, the filaments will each:

- carry current that may vary in time;

- be free to move axisymmetrically;

- have finite mass and resistance that are assumed constant.

\section{A. Energy Functions}

In the next phase of the modeling process, we will select the generalized coordinates and introduce the external voltages into the potential energy function.

Consistent with the modeling assumptions, we define the generalized coordinates $\boldsymbol{q}$ via

$$
\dot{\boldsymbol{q}}=\left[\begin{array}{c}
\boldsymbol{I}_{e} \\
\boldsymbol{I}_{s} \\
\dot{\boldsymbol{r}}
\end{array}\right]
$$

The vector $\boldsymbol{I}_{e}$ contains the currents in the plasma elements, $\boldsymbol{I}_{s}$ is a vector of structure currents and $\boldsymbol{r}=\left[\begin{array}{l}\boldsymbol{R} \\ \boldsymbol{z}\end{array}\right]$ is a vector of position coordinates associated with the plasma current elements.

Next, we introduce the inductance-mass matrix

$$
\mathbf{T}(\boldsymbol{q})=\left[\begin{array}{ccc}
\mathbf{L}_{e} & \mathbf{M}_{e s} & 0 \\
\mathbf{M}_{e s}^{\prime} & \mathbf{L}_{s} & 0 \\
0 & 0 & \mathbf{m}_{e}
\end{array}\right]
$$

in which $\mathbf{L}$ and $\mathbf{M}$ are the self and mutual inductance matrices. Again, subscript $e$ denotes the plasma elements and subscript $s$ denotes the structure elements. The matrices $\mathbf{L}_{e}$ and $\mathbf{M}_{e s}$ are functions of plasma element position, because the plasma current elements are free to move axisymmetrically. The structure self-inductance matrix, $\mathbf{L}_{s}$, is constant. Note that $\mathbf{M}_{s e}=\mathbf{M}_{e s}^{\prime}$. The constant diagonal mass matrix, $\mathbf{m}_{e}$, contains the mass of each plasma current element.

The input vector is given by

$$
\boldsymbol{U}=\left[\begin{array}{c}
\boldsymbol{V}_{e} \\
\boldsymbol{V}_{s} \\
0
\end{array}\right]
$$

where $\boldsymbol{V}_{e}$ is a vector of effective voltages applied to each element (for example, by ion injection) and $\boldsymbol{V}_{s}$ is the vector of externally applied poloidal field coil voltages.
The resistance matrix is defined as

$$
\boldsymbol{\Omega}=\left[\begin{array}{ccc}
\boldsymbol{\Omega}_{e} & 0 & 0 \\
0 & \boldsymbol{\Omega}_{s} & 0 \\
0 & 0 & 0
\end{array}\right]
$$

The generalized kinetic energy is given by

$$
\mathcal{T}=\frac{1}{2} \dot{\boldsymbol{q}}^{\prime} \mathbf{T} \dot{\boldsymbol{q}}
$$

and the generalized potential is

$$
\mathcal{V}=-W-q^{\prime} \boldsymbol{U}+\frac{1}{2} \frac{d\left(\int_{\boldsymbol{q}\left(t_{0}\right)}^{\boldsymbol{q}\left(t_{0}\right.} \int_{\boldsymbol{q}\left(t_{0}\right)}^{\boldsymbol{q}(t)} d \boldsymbol{q}^{\prime} \boldsymbol{\Omega} d \boldsymbol{q}\right)}{d t}
$$

in which $W$ represents the plasma's internal energy.

As usual, the Lagrangian is given by

$$
\mathcal{L}=\frac{1}{2} \dot{\boldsymbol{q}}^{\prime} \mathbf{T} \dot{\boldsymbol{q}}+W+\boldsymbol{q}^{\prime} \boldsymbol{U}-\frac{1}{2} \frac{d\left(\int_{\boldsymbol{q}\left(t_{0}\right)}^{\boldsymbol{q}(t)} \int_{\boldsymbol{q}\left(t_{0}\right)}^{\boldsymbol{q}(t)} d \boldsymbol{q}^{\prime} \boldsymbol{\Omega} d \boldsymbol{q}\right)}{d t} .
$$

The term containing $\Omega$ is the total energy dissipated from time $t_{0}$ to $t$; it is essentially an integral form of Ohm's law. In the Hamiltonian formulation, the dissipation term must be included in $\mathcal{V}$ and not as a separate power dissipation function.

\section{B. Plasma Internal Energy}

We will see later that the internal energy $W$ varies as $\boldsymbol{R} \boldsymbol{I}_{e}^{2}$. As such, we can express it as

$$
W=\frac{\boldsymbol{q}^{\prime} \mathbf{E} \dot{\boldsymbol{q}}^{2}}{2}
$$

where $\mathbf{E}$ is a constant matrix. The $\dot{\boldsymbol{q}}^{2}$ notation refers to a vector containing quadratic terms in each entry (the elemental currents in this case).

This representation is chosen for convenience when forming the Hamiltonian.

\section{Equations of Motion}

Using the previous information, we can now express the Lagrangian (3) as

$$
\begin{aligned}
\mathcal{L}=\frac{1}{2} \dot{\boldsymbol{q}}^{\prime} \mathbf{T} \dot{\boldsymbol{q}}+\frac{\boldsymbol{q}^{\prime} \mathbf{E} \dot{\boldsymbol{q}}^{2}}{2}+\boldsymbol{q}^{\prime} \boldsymbol{U} & \\
& -\frac{1}{2} \frac{d\left(\int_{\boldsymbol{q}\left(t_{0}\right)}^{\boldsymbol{q}(t)} \int_{\boldsymbol{q}\left(t_{0}\right)}^{\boldsymbol{q}(t)} d \boldsymbol{q}^{\prime} \boldsymbol{\Omega} d \boldsymbol{q}\right)}{d t} .
\end{aligned}
$$

To form the Hamiltonian, via the correct Legendre transformation, the generalized momenta are computed next

$$
\boldsymbol{p}=\frac{\partial \mathcal{L}}{\partial \dot{q}}
$$

giving

$$
p=\mathbf{T} \dot{q}+q^{\prime} \mathbf{E} \dot{\boldsymbol{q}}
$$


The Hamiltonian is given by

$$
\mathcal{H}=p^{\prime} \dot{\boldsymbol{q}}-\mathcal{L}
$$

or equivalently

$$
\begin{aligned}
& \mathcal{H}=\frac{1}{2} \dot{\boldsymbol{q}}^{\prime} \mathbf{T} \dot{\boldsymbol{q}}+\frac{1}{2} \boldsymbol{q}^{\prime} \mathbf{E} \dot{\boldsymbol{q}}^{2}-\boldsymbol{q}^{\prime} \mathbf{U} \\
&+\frac{1}{2} \frac{d\left(\int_{\boldsymbol{q}\left(t_{0}\right)}^{\boldsymbol{q}(t)} \int_{\boldsymbol{q}\left(t_{0}\right)}^{\boldsymbol{q}(t)} d \boldsymbol{q}^{\prime} \boldsymbol{\Omega} d \boldsymbol{q}\right)}{d t} .
\end{aligned}
$$

We now eliminate $\dot{\boldsymbol{q}}$ using (6), to obtain the Hamiltonian in terms of $\boldsymbol{p}, \boldsymbol{q}$, and $t$

$$
\begin{aligned}
\mathcal{H}(\boldsymbol{p}, \boldsymbol{q}, t)=\frac{1}{2} \boldsymbol{p}^{\prime}[\mathbf{T}+\boldsymbol{q} \mathbf{E}]^{-1} \boldsymbol{p}-\boldsymbol{q}^{\prime} \boldsymbol{U} \\
+\frac{1}{2} \frac{d\left(\int_{\boldsymbol{q}\left(t_{0}\right)}^{\boldsymbol{q}(t)} \int_{\boldsymbol{q}\left(t_{0}\right)}^{\boldsymbol{q}(t)} d \boldsymbol{q}^{\prime} \boldsymbol{\Omega} d \boldsymbol{q}\right)}{d t} .
\end{aligned}
$$

The relation

$$
\dot{p}=-\frac{\partial \mathcal{H}}{\partial q}
$$

gives

$$
\begin{aligned}
& \frac{d\left(\mathbf{T} \dot{\boldsymbol{q}}+\boldsymbol{q}^{\prime} \mathbf{E} \dot{\boldsymbol{q}}+\boldsymbol{\Omega q}\right)}{d t} \\
& =\boldsymbol{U}+\frac{\mathbf{E} \dot{\boldsymbol{q}}^{2}}{2}+\frac{1}{2} \dot{\boldsymbol{q}}^{\prime} \frac{\partial \mathbf{T}}{\partial \boldsymbol{q}} \dot{\boldsymbol{q}}-\frac{1}{2} \frac{d\left(\int_{\boldsymbol{q}\left(t_{0}\right)}^{\boldsymbol{q}(t)} d \boldsymbol{q}^{\prime} \boldsymbol{\Omega}\right)}{d t}
\end{aligned}
$$

which is the required equation of motion.

This equation can now be expanded into four vector equations. The equation for $\boldsymbol{I}_{e}$ is

$$
\frac{d\left(\mathbf{L}_{e} \boldsymbol{I}_{e}+\mathbf{M}_{e s} \boldsymbol{I}_{s}+\boldsymbol{R} \mathbf{E} \boldsymbol{I}_{e}\right)}{d t}+\boldsymbol{\Omega}_{e} \boldsymbol{I}_{e}=\boldsymbol{V}_{e}
$$

which is essentially a statement of Kirchoff's voltage law for the plasma elements.

Similarly, we have Kirchoff's voltage law for the structural and poloidal circuits, giving the equation for $\boldsymbol{I}_{s}$

$$
\frac{d\left(\mathbf{L}_{s} \boldsymbol{I}_{s}+\mathbf{M}_{s e} \boldsymbol{I}_{e}\right)}{d t}+\boldsymbol{\Omega}_{s} \boldsymbol{I}_{s}=V_{s}
$$

The remaining equations are force balances in the $R$ and $z$-directions, respectively. For $\boldsymbol{R}$, we have

$$
\frac{d\left(\mathbf{m}_{e} \dot{\boldsymbol{R}}\right)}{d t}=\frac{1}{2} \boldsymbol{I}_{e}^{\prime} \frac{\partial \mathbf{L}_{e}}{\partial \boldsymbol{R}} \boldsymbol{I}_{e}+\boldsymbol{I}_{s}^{\prime} \frac{\partial \mathbf{M}_{s e}}{\partial \boldsymbol{R}} \boldsymbol{I}_{e}+\frac{\mathbf{E} \boldsymbol{I}_{e}^{2}}{2}
$$

and for $z$

$$
\frac{d\left(\mathbf{m}_{e} \dot{\boldsymbol{z}}\right)}{d t}=\frac{1}{2} \boldsymbol{I}_{e}^{\prime} \frac{\partial \mathbf{L}_{e}}{\partial \boldsymbol{z}} \boldsymbol{I}_{e}+\boldsymbol{I}_{s}^{\prime} \frac{\partial \mathbf{M}_{s e}}{\partial \boldsymbol{z}} \boldsymbol{I}_{e}
$$

We conclude this section with a number of observations.
- In the case that $\mathcal{H}$ is independent of a particular coordinate, the corresponding canonical momentum will be conserved (see (9), and by Noether's theorem [16, Sec. 12-7]. In the same way, if $\mathcal{H}$ is time invariant, the associated system is conservative.

- If $\boldsymbol{U}$ and $\boldsymbol{\Omega}$ are both zero, the magnetic fluxes $\boldsymbol{p}_{e}$ and $\boldsymbol{p}_{s}$ will remain constant. This situation is analogous to a system of purely inductive closed loops. More specifically $\boldsymbol{V}_{e}=0, \boldsymbol{\Omega}_{e}=0$ implies constancy of the magnetic flux associated with the plasma model. These assumptions result in the well-known ideal magneto-hydrodynamic (MHD) situation, in which lines of constant flux are "frozen" in the plasma. Since some plasma resistance will occur in practice, the $\boldsymbol{\Omega}_{e}=0$ assumption is generally false. A distinguishing feature of the model we present is the introduction and consideration of resistance terms in the plasma model from the beginning.

- Replacing the generalized coordinates with $-\boldsymbol{p}$ gives an equivalent model, because of the invariance properties of the canonical equations. In the case of a nonresistive plasma, one may use the fluxes associated with the plasma elements as generalized coordinates. Since one cannot express the resistive form of Ohm's law in terms of flux, this necessitates a loss of generality. As a result, plasma models that describe plasma behavior in terms of plasma flux functions must necessarily neglect plasma resistance effects.

- Also evident from the $\boldsymbol{I}_{e}$ equation is the necessity of current ramping in $\boldsymbol{I}_{s}$ to maintain a steady $\boldsymbol{I}_{e}$ against the plasma resistance, or alternatively a nonzero $V_{e}$. It can be seen from (12), that in the case of a plasma resistance, a constant $\boldsymbol{V}_{s}$ will not suffice to maintain a steady plasma current and position. As such, the plasma resistance is an important feature of the model. This conclusion is illustrated by a simple, intuitive example in the Appendix.

- It is interesting to note that as the plasma mass approaches zero, the plasma profile adjusts to the fields instantaneously. This is the approximation of instantaneous MHD equilibrium.

\section{Definitions of Plasma Bulk Properties}

A lumped-parameter model can be defined from (11) to (14), by defining various averaged plasma quantities. The total plasma current will be called $I_{p}$. The equilibrium plasma current density distribution $j(R, z)$ is calculated from the Grad-Shafranov equation [1], [2] by an inverse equilibrium reconstruction code. We take the plasma mass to be zero, because the inertial forces are tiny compared to the other forces experienced by the plasma.

The average plasma radial position $R$ is defined by a currentweighted average of plasma element radial positions [17]

$$
R=\frac{\sum_{k} i_{k} R_{k}}{\sum_{k} i_{k}}
$$


The average plasma vertical position $z$ is defined similarly.

The effective mutual inductance matrix between the plasma and structure $\mathbf{M}_{p s}$ is

$$
I_{p} \mathbf{M}_{p s} \boldsymbol{I}_{s}=\sum_{k} i_{k} \mathbf{M}_{k s} \boldsymbol{I}_{s}
$$

where $\mathbf{M}_{k s}$ is the mutual inductance between the $k$ th plasma element and the vector of structure element currents.

We define the effective plasma self inductance $L_{p}$ via the equivalent energy of the total current distribution

$$
\frac{1}{2} L_{p} I_{p}^{2}=\frac{1}{2} \sum_{k} \sum_{h} i_{k} M_{k h} i_{h}
$$

where $M_{k h}$ is the mutual inductance between the $h$ th and $k$ th elements, for $h \neq k$. In the case of $h=k$, the self inductance of the $k$ th element is used.

A lumped-parameter model can be defined from (11) to (14), by defining various averaged plasma quantities. The total plasma current will be called $I_{p}$. The equilibrium plasma current density distribution $j(R, z)$ is calculated from the Grad-Shafranov equation [1], [2] by an inverse equilibrium reconstruction code. We take the plasma mass to be zero, because the associated modes would operate on a frequency much higher than the range of interest.

The average plasma radial position $R$ is defined by a currentweighted average of plasma element radial positions [17]

$$
R=\frac{\sum_{k} i_{k} R_{k}}{\sum_{k} i_{k}}
$$

The average plasma vertical position $z$ is defined similarly.

The effective mutual inductance matrix between the plasma and structure $\mathbf{M}_{p s}$ is

$$
I_{p} \mathbf{M}_{p s} \boldsymbol{I}_{s}=\sum_{k} i_{k} \mathbf{M}_{k s} \boldsymbol{I}_{s}
$$

where $\mathbf{M}_{k s}$ is the mutual inductance between the $k$ th plasma element and the vector of structure element currents.

We define the effective plasma self inductance $L_{p}$ via the equivalent energy of the total current distribution

$$
\frac{1}{2} L_{p} I_{p}^{2}=\frac{1}{2} \sum_{k} \sum_{h} i_{k} M_{k h} i_{h}
$$

where $M_{k h}$ is the mutual inductance between the $h$ th and $k$ th elements, for $h \neq k$. In the case of $h=k$, the self inductance of the $k$ th element is used.

To evaluate the internal energy of the plasma, $W$, we may start with the equations governing the plasma profile

$$
\begin{gathered}
\nabla p=\boldsymbol{j} \times \boldsymbol{B} \\
\mu_{0} \boldsymbol{j}=\nabla \times \boldsymbol{B} .
\end{gathered}
$$

By substitution we have

$$
\mu_{0} \nabla p=(\nabla \times \boldsymbol{B}) \times \boldsymbol{B} .
$$

Using the identity

$$
(\nabla \times A) \times A=(A \cdot \nabla) A-\frac{1}{2} \nabla A^{2}
$$

gives

$$
\begin{aligned}
\mu_{0} \nabla p & =(\boldsymbol{B} \cdot \nabla) \boldsymbol{B}-\frac{1}{2} \nabla \boldsymbol{B}^{2} \\
& \Rightarrow \nabla\left(p+\frac{\boldsymbol{B}^{2}}{2 \mu_{0}}\right)=(\boldsymbol{B} \cdot \nabla) \boldsymbol{B} .
\end{aligned}
$$

At equilibrium and for our geometry the right-hand side is small (zero for the circular, small aspect ratio approximation), which gives

$$
p+\frac{\boldsymbol{B}^{2}}{2 \mu_{0}}=\text { const. }=\frac{\boldsymbol{B}_{0}^{2}}{2 \mu_{0}}
$$

where $\boldsymbol{B}_{0}$ is the magnetic field outside the plasma (where $p=$ $0)$.

We define

$$
\beta=\frac{p}{\left(\frac{B^{2}}{2 \mu_{0}}\right)} .
$$

Since $W$ is the energy associated with the pressure, and taking the plasma volume $V$ as $V=2 \pi R S$ where $S$ is the plasma cross-sectional area, we have for $W$

$$
W=p V=\frac{\boldsymbol{B}^{2}}{2 \mu_{0}} \beta S 2 \pi R
$$

We can write

$$
W=p V=\int p d S 2 \pi R .
$$

Defining an average poloidal field as $\overline{\boldsymbol{B}}_{\theta}=\left(\left(\mu_{0} I_{p}\right) /(l)\right)$, gives a correspondingly averaged poloidal beta

$$
\beta_{p}=\frac{\int p d S}{S} \frac{2 \mu_{0}}{\bar{B}_{\theta}^{2}} .
$$

We can then approximate $W$ as

$$
\begin{aligned}
W & =\frac{\overline{\boldsymbol{B}}_{\theta}^{2}}{2 \mu_{0}} \beta_{p} S 2 \pi R . \\
& =\mu_{0} \pi \frac{S}{l^{2}} \beta_{p} R I_{p}^{2} .
\end{aligned}
$$

The proper evaluation of $W$ requires careful consideration. For comparison, a slightly different treatment is to be found in [9]. 


\section{E. Linearization of Equations}

Equations (11)-(14) define the evolution of the variables $\left(R, z, I_{p}, I_{s}\right)$. For consistency with [6], we will replace the variables $(R, z)$ with $\left(R I_{p}^{0}, z I_{p}^{0}\right)$ in which $I_{p}^{0}$ is the constant equilibrium plasma current. With this change of variable in place, we introduce the perturbations

$$
\boldsymbol{x}=\left[\begin{array}{c}
\boldsymbol{I}_{s}-\boldsymbol{I}_{s}^{0} \\
\left(z-z^{0}\right) I_{p}^{0} \\
\left(R-R^{0}\right) I_{p}^{0} \\
I_{p}-I_{p}^{0}
\end{array}\right]=\left[\begin{array}{c}
\delta \boldsymbol{I}_{s} \\
\delta z I_{p}^{0} \\
\delta R I_{p}^{0} \\
\delta I_{p}
\end{array}\right] .
$$

The four physics equations are therefore linearized in $x$ about the tokamak equilibrium $x^{0}=0$, to give four linear equations. These linear matrices can then be cast in the standard state-space model form

$$
\begin{aligned}
& \dot{x}=\mathbf{A} x+\mathbf{B} u \\
& y=\mathbf{C} x+\mathbf{D} u
\end{aligned}
$$

in which $\boldsymbol{x}$ is an $n$-dimensional state vector, $\mathbf{A}$ is an $n \times n$ real-valued matrix, $\mathbf{B}$ is an $n \times r$ real-valued matrix, $\boldsymbol{u}$ is an $r$-vector of control inputs, $\boldsymbol{y}$ is a $p$-vector of outputs, $\mathbf{C}$ is a $p \times n$ real-valued matrix and $\mathbf{D}$ is a $p \times r$ real-valued matrix.

The linearized structure circuit equation, plasma circuit equation and plasma force balance equations can be represented as shown in (23) at the bottom of the page.

This is of the form $\mathcal{M} \dot{\boldsymbol{x}}+\mathcal{R} \boldsymbol{x}=\boldsymbol{u}$. Comparing (23) with (22) gives the matrix of coefficients $\mathbf{A}$, and the control matrix $\mathbf{B}$

$$
\begin{aligned}
& \mathbf{A}=-\mathcal{M}^{-1} \mathcal{R} \\
& \mathbf{B}=\mathcal{M}^{-1}\left[\begin{array}{llll}
\mathbf{I} & \mathbf{0} & \mathbf{0} & \mathbf{0} \\
0 & 0 & 0 & I
\end{array}\right]^{T} .
\end{aligned}
$$

Note that $\mathcal{M}$ and $\mathcal{R}$ are symmetric with $\mathcal{M}$ positive-definite and $\mathcal{R}$ positive-semidefinite.

From a minimal set of assumptions we have derived a linear, time invariant model in state-space form. All linearized tokamak models making similar assumptions can be expressed in this structural form. Within this framework we can derive all models that represent axisymmetric perturbations about given MHD equilibria.

\section{F. RZIP Linear Model}

Further to the assumptions detailed earlier, the linear RZIP model also makes the rigid current displacement assumption, namely that the normalized current profile is independent of movements in the $R$ and $z$ directions and of changes in plasma current. It follows therefore that changes in the plasma current profile are not modeled. As such, $\left.\left(\left(\partial L_{p}\right) /(\partial z)\right)\right|_{0}=0$.

Changes to the current and pressure profile can be approximately modeled by permitting perturbations to $\beta_{p}$. These changes are then introduced as disturbances in $\dot{\beta}_{p}$ on the right-hand side of (23), as in [9].

The rigid current displacement assumption allows us to calculate the plasma mutual and self inductance derivatives simply and directly.

For example, the mutual inductance between two plasma elements must satisfy

$$
\frac{\partial M_{f g}}{\partial R}=\frac{\partial M_{f g}}{\partial R_{f}} \frac{\partial R_{f}}{\partial R}+\frac{\partial M_{f g}}{\partial R_{g}} \frac{\partial R_{g}}{\partial R} .
$$

The rigid current displacement assumption fixes the two radius relations as

$$
\frac{\partial R_{f}}{\partial R}=\frac{\partial R_{g}}{\partial R}=1
$$

\section{CONTROL PROBLEM}

The remainder of the paper is concerned with the design and evaluation of a control system for the TCV tokamak [15]. We will use the linearized model described in Section II as a basis for designing this controller. It will be tested on a nonlinear code based on a sequence of Grad-Shafranov equilibria [18].

\section{A. Control Objectives}

The primary interest of this work is the study of control systems for elongated plasmas in TCV. As with all elongated plasmas, the equilibrium under consideration is unstable in the vertical position and so the open-loop plant is characterized by a single unstable pole that requires stabilization. One of the major challenges is the uncertainty in the modeling of the tokamak plasma dynamics together with the fact that these dynamics vary with the operating conditions. For this reason,

$$
\begin{aligned}
& {\left[\begin{array}{cccc}
\mathbf{L}_{s} & \left.\frac{\partial \mathbf{M}_{s p}}{\partial z}\right|_{0} & \left.\frac{\partial \mathbf{M}_{s p}}{\partial R}\right|_{0} & \mathbf{M}_{s p}^{0} \\
\left.\frac{\partial \mathbf{M}_{p s}}{\partial z}\right|_{0} & \left(\left.\frac{1}{2} \frac{\partial^{2} L_{p}}{\partial z^{2}}\right|_{0}+\left.\frac{\partial^{2} \mathbf{M}_{p s}}{\partial z^{2}}\right|_{0} \frac{\boldsymbol{I}_{s}^{0}}{I_{p}^{0}}\right) & \left(\left.\frac{1}{2} \frac{\partial^{2} L_{p}}{\partial z \partial R}\right|_{0}+\left.\frac{\partial^{2} \mathbf{M}_{p s}}{\partial z \partial R}\right|_{0} \frac{\boldsymbol{I}_{s}^{0}}{I_{p}^{0}}\right) & \left.\frac{\partial L_{p}}{\partial z}\right|_{0} \\
\left.\frac{\partial \mathbf{M}_{p s}}{\partial R}\right|_{0} & \left(\left.\frac{1}{2} \frac{\partial^{2} L_{p}}{\partial z \partial R}\right|_{0}+\left.\frac{\partial^{2} \mathbf{M}_{p s}}{\partial z \partial R}\right|_{0} \frac{\boldsymbol{I}_{s}^{0}}{I_{p}^{0}}\right) & \left(\left.\frac{1}{2} \frac{\partial^{2} L_{p}}{\partial R^{2}}\right|_{0}+\left.\frac{\partial^{2} \mathbf{M}_{p s}}{\partial R^{2}}\right|_{0} \frac{\boldsymbol{I}_{s}^{0}}{I_{p}^{0}}\right) & \left.\left(\left.\frac{\partial L_{p}}{\partial R}\right|_{0}+\left.\frac{\partial \mathbf{M}_{p s}}{\partial R}\right|_{0} \frac{\boldsymbol{I}_{s}^{0}}{I_{p}^{0}}+\mu_{0} \frac{2 \pi S}{l^{2}} \beta_{p}\right)\right|_{0} \\
\mathbf{M}_{p s}^{0} & \left.\frac{\partial L_{p}}{\partial z}\right|_{0} & \left(\left.\frac{\partial \mathbf{M}_{p s}}{\partial R}\right|_{0} \frac{\boldsymbol{I}_{s}^{0}}{I_{p}^{0}}+\left.\frac{\partial L_{p}}{\partial R}\right|_{0}+\mu_{0} \frac{2 \pi S}{l^{2}} \beta_{p}\right) & L_{p}^{0}+\mu_{0} \frac{2 \pi S}{l^{2}} \beta_{p} R^{0}
\end{array}\right]} \\
& +\left[\begin{array}{cccc}
\boldsymbol{\Omega}_{\mathbf{s}} & 0 & 0 & 0 \\
\mathbf{0} & 0 & 0 & 0 \\
\mathbf{0} & 0 & 0 & 0 \\
\mathbf{0} & 0 & 0 & \Omega_{p}
\end{array}\right] \boldsymbol{x}=\left[\begin{array}{ll}
\mathbf{I} & 0 \\
\mathbf{0} & 0 \\
\mathbf{0} & 0 \\
\mathbf{0} & I
\end{array}\right]\left[\begin{array}{l}
\delta \mathbf{V}_{s} \\
\delta V_{p}
\end{array}\right] .
\end{aligned}
$$




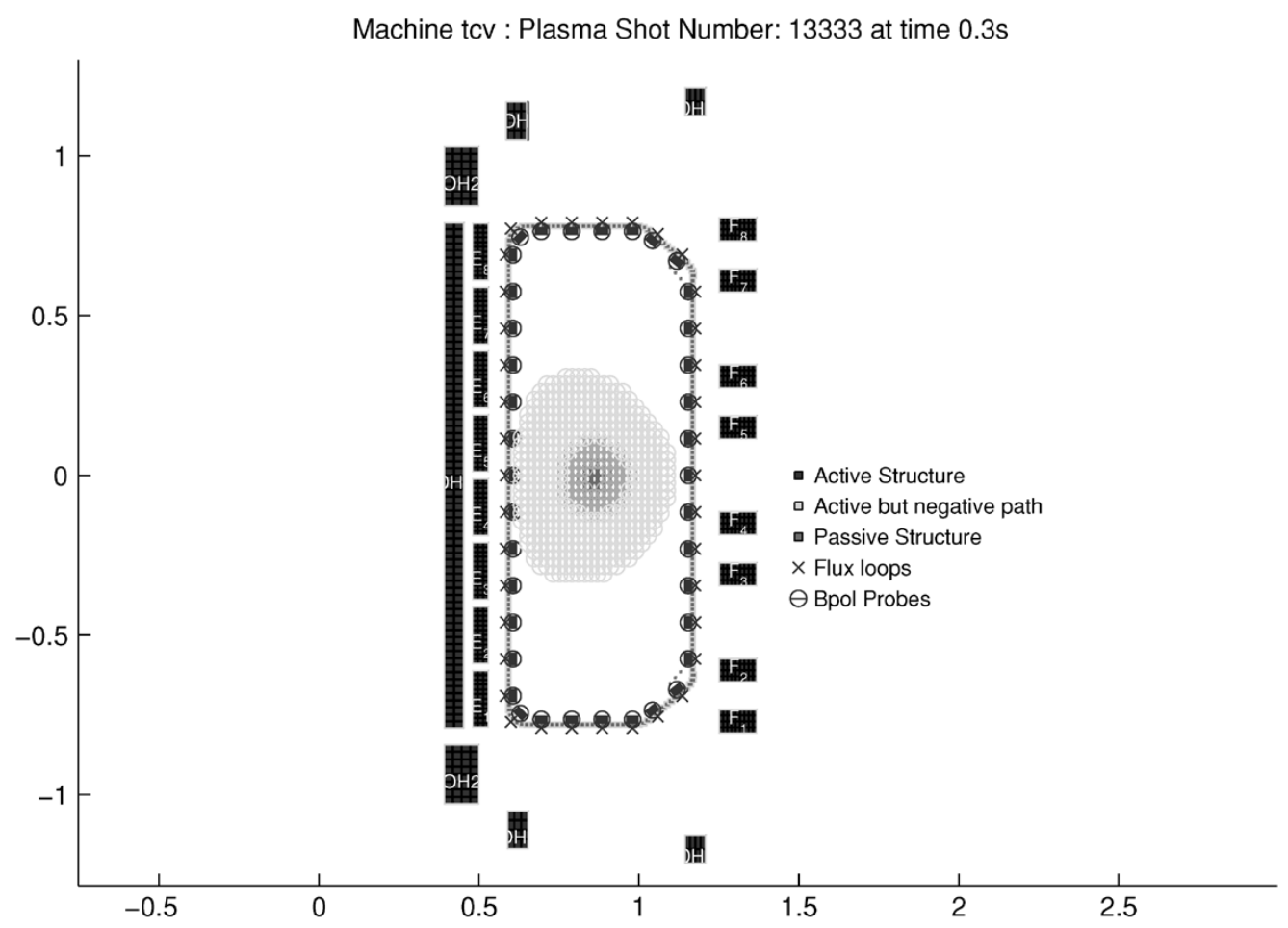

Fig. 4. TCV equilibrium 13333.

it is important that the proposed control system has adequate robust stability and performance margins. We will also seek to decouple the five outputs that characterize the plasma shape, position and current. Ideally, we would like to be able to implement variation in any one of these outputs without influencing the others.

\section{B. RZIP Model of TCV}

The RZIP model plus power supplies has been qualified against the TCV equilibrium 13333 [6]. The plasma in this shot is well understood and unstable with a moderate growth rate measured and predicted by RZIP as approximately $100 \mathrm{~s}^{-1}$; the equilibrium profile is shown in Fig. 4. The five control parameters used for controlling this type of equilibrium on TCV are:

- $\quad p_{\text {vert }}$, the radial flux imbalance (a radial position measurement);

- $\quad \operatorname{tri}_{\text {out }}$, the outboard field curvature;

- $\quad \operatorname{tri}_{\text {in }}$, the inboard field curvature;

- $z I_{p}$, the product of plasma vertical position and current;

- $I_{p}$, the plasma current.

The particular RZIP model used for controller design assumes a superconducting plasma (see Section II). This choice is made because the controller will be tested on the ideal MHD code PROTEUS, which makes a similar assumption. Fig. 5 shows the singular value plot of the TCV RZIP model with 18 inputs and five outputs. It can be seen from this figure that there is a large spread in the singular value magnitudes. As is standard practice, we will seek to reduce the transfer function's

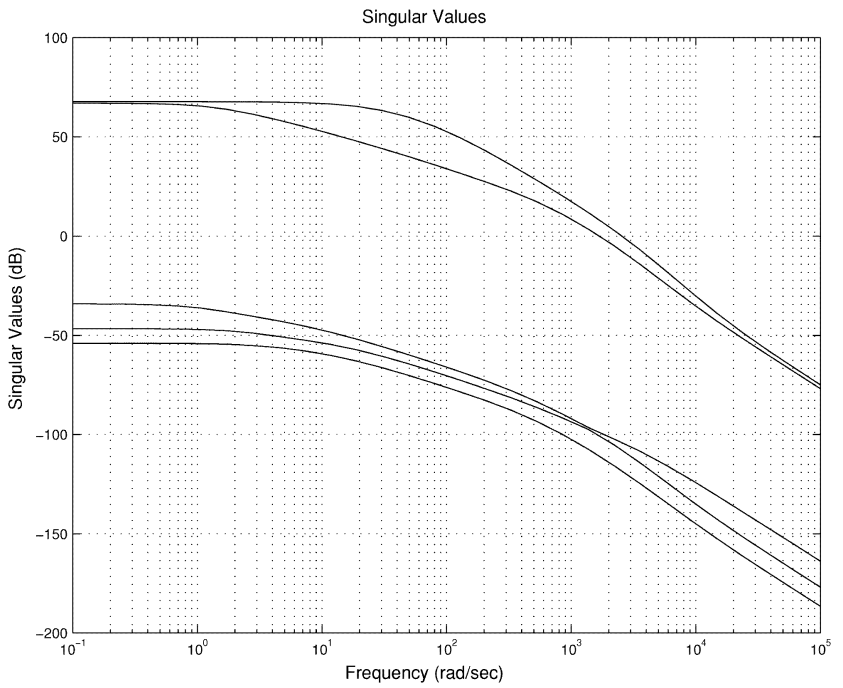

Fig. 5. Singular value plot of TCV RZIP model.

condition number by scaling. Indeed, this will be the initial step of a loop shaping design.

A characterizing feature of models based on ideal MHD assumptions is the introduction of uncontrollable modes at the origin. The underlying mechanism that gives rise to these modes is described briefly in the Appendix using simple circuit theoretic arguments. Since these poles cannot exist in reality, we will remove them from the RZIP model using a standard model reduction algorithm [19]. 


\section{Modeling of Power Supplies}

The poloidal field coil power supplies are approximated by single pole filters, expressed in the state-space form as

$$
\begin{aligned}
& A=-\operatorname{diag}\left(\frac{1}{\tau_{c}}\right) \\
& B=\operatorname{diag}\left(\frac{1}{\tau_{c}}\right) \\
& C=I \\
& D=I
\end{aligned}
$$

where $\tau_{c}=0.3 \mathrm{~ms}$ for all PF coil power supplies used. Throughout the plant referred to is the tokamak including the power supplies.

\section{Robust CONTROLler DESIGN}

Motivated by its simplicity, we will use the the $\mathcal{H}_{\infty}$ normalized coprime factorization method [20], [21] for the controller synthesis. In many respects, the research presented here is an evolution of that presented in [22]. After completing the loop shaping and controller synthesis, we will make use of some recent research [14] that facilitates a reduction in the controller order with guaranteed properties.

\section{A. Normalized Left Coprime Factorization Perturbations}

A set of plant models that will be used to represent the machine will be characterized in terms if a perturbed normalized coprime factorization. We suppose that $\mathbf{G}$ is the nominal plant model. Then

$$
\mathrm{G}=\mathrm{M}^{-1} \mathbf{N}
$$

is a normalized left coprime factorization of $\mathbf{G}$ if $\mathbf{M}, \mathbf{N} \in \mathcal{H}_{\infty}$ are coprime and satisfy

$$
\mathrm{MM}^{\prime}+\mathrm{NN}^{\prime}=\mathbf{I}
$$

Given such a factorization, we define the model set

$$
\begin{aligned}
\mathcal{G}_{\gamma}=\left\{\left(\mathbf{M}-\boldsymbol{\Delta}_{M}\right)^{-1}\left(\mathbf{N}+\boldsymbol{\Delta}_{N}\right):\left[\boldsymbol{\Delta}_{N} \boldsymbol{\Delta}_{M}\right] \in \mathcal{H}_{\infty}\right. \\
\left.\times\left\|\left[\boldsymbol{\Delta}_{N} \boldsymbol{\Delta}_{M}\right]\right\|_{\infty}<\gamma^{-1}\right\}
\end{aligned}
$$

\section{B. Optimization Problem}

Our aim is to design a controller that is optimal in the sense of minimizing $\gamma$, in order to maximize the size of admissible perturbations [by (25)]. From Fig. 6 we see that

$$
\left[\begin{array}{l}
u \\
y
\end{array}\right]=\mathcal{H}(\mathbf{G}, \mathbf{K}) \phi, \quad \phi=\left[\begin{array}{ll}
\boldsymbol{\Delta}_{N} & \boldsymbol{\Delta}_{M}
\end{array}\right]\left[\begin{array}{l}
u \\
y
\end{array}\right]
$$

where

$$
\mathcal{H}(\mathbf{G}, \mathbf{K})=\left[\begin{array}{c}
\mathbf{K} \\
\mathbf{I}
\end{array}\right](\mathbf{I}-\mathbf{G K})^{-1} \mathbf{M}^{-1}
$$

From the small gain theorem [20], the condition

$$
\|\mathcal{H}(\mathbf{G}, \mathbf{K})\|_{\infty} \leq \gamma
$$

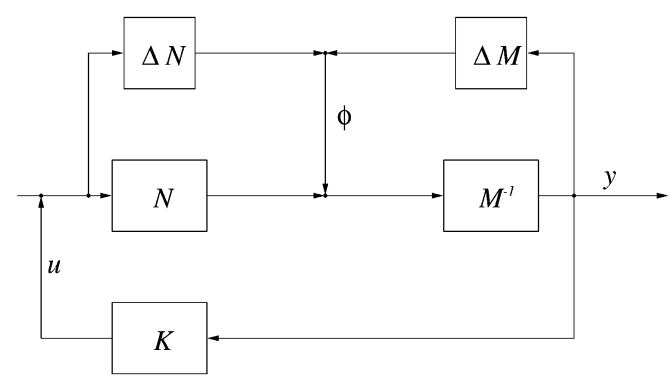

Fig. 6. Normalized left-coprime optimal controller design problem.

will ensure that the closed loop will be stable for all plants in the model set $\mathcal{G}_{\gamma}$. To maximize the robustness of the closed loop, we require a controller that minimizes $\gamma$.

\section{Parameterization of All Suboptimal Controllers}

The following result gives a parameterization of all suboptimal controllers.

Lemma IV.1: [14], [20], [21], [23]: Let $\mathbf{G}$ have a minimal realization $\mathbf{G} \stackrel{s}{=}(A, B, C, 0)$. Then there exist unique stabilizing and positive-definite solutions $X, Y$ to the algebraic Riccati equations

$$
\begin{aligned}
A^{\prime} X+X A-X B B^{\prime} X+C^{\prime} C & =0 \\
A Y+Y A^{\prime}-Y C^{\prime} C Y+B B^{\prime} & =0
\end{aligned}
$$

respectively, and

$$
\gamma_{\mathrm{opt}}=\sqrt{1+\lambda_{\max }(X Y)} \geq 1
$$

For any $\gamma>\gamma_{\text {opt }} \geq 1$ let $\beta=\sqrt{1-\gamma^{-2}}$ so that $0<\beta \leq 1$ and define $Z=\left(I-\gamma^{-2} \beta^{-2} X Y\right)^{-1}$. Then all suboptimal controllers are given by the parametrization

$$
\begin{array}{r}
\mathbf{K}=\left(\boldsymbol{\Theta}_{11} \mathbf{Q}+\boldsymbol{\Theta}_{12}\right)\left(\boldsymbol{\Theta}_{21} \mathbf{Q}+\boldsymbol{\Theta}_{22}\right)^{-1} \\
\mathbf{Q} \in \mathcal{R} \mathcal{H}_{\infty}, \quad\|\mathbf{Q}\|_{\infty}<\gamma
\end{array}
$$

where

$$
\begin{aligned}
\boldsymbol{\Theta} & =\left[\begin{array}{ll}
\boldsymbol{\Theta}_{11} & \boldsymbol{\Theta}_{12} \\
\boldsymbol{\Theta}_{21} & \boldsymbol{\Theta}_{22}
\end{array}\right] \\
& \stackrel{s}{=}\left[\begin{array}{c|cc}
A-B B^{\prime} X & Z B & \beta^{-1} Z Y C^{\prime} \\
\hline-\beta^{-2} B^{\prime} X & I & 0 \\
\beta^{-2} C & 0 & \beta^{-1} I
\end{array}\right] \in \mathcal{R} \mathcal{H}_{\infty} .
\end{aligned}
$$

We will also require

$$
\boldsymbol{\Theta}^{-1} \stackrel{s}{=}\left[\begin{array}{c|cc}
Z\left(A-Y C^{\prime} C\right) Z^{-1} & Z B & Z Y C^{\prime} \\
\hline \beta^{-2} B^{\prime} X & I & 0 \\
-\beta^{-1} C & 0 & \beta I
\end{array}\right] \in \mathcal{R} \mathcal{H}_{\infty}
$$

in the sequel. 
Remark IV.1: Taking $\mathbf{Q}=0$ in (31) gives the central controller in the form of a right coprime factorization

$$
\begin{aligned}
\mathbf{K}_{11} & =\boldsymbol{\Theta}_{12} \boldsymbol{\Theta}_{22}^{-1} \\
& \stackrel{s}{=}\left[\begin{array}{c|c}
A-B B^{\prime} X-\beta^{-2} Z Y C^{\prime} C & Z Y C^{\prime} \\
\hline-\beta^{-2} B^{\prime} X & 0
\end{array}\right] .
\end{aligned}
$$

We will use this controller throughout.

\section{Choice of Weighting Matrices}

It is evident from Fig. 5 that the unweighted plant model has five nonzero singular values spread over at least $100 \mathrm{~dB}$ at low frequency. As this will lead to highly "direction sensitive" responses, it was deemed necessary to rescale the outputs, thereby balancing the singular values. To do this, we postmultiply $\mathbf{G}$ by

$$
\mathbf{W}_{o}=500 \mathbf{G}^{\#}(0)
$$

to give

$$
\mathbf{G}_{W}=500 \mathbf{G}^{\#}(0) \mathbf{G} .
$$

The singular values of the weighted plant $\mathbf{G}_{W}$ are shown in Fig. 7. As expected, five of the singular values have associated DC gain of $500(54 \mathrm{~dB})$, while the remaining ones are effectively zero. As will be shown, this simple loop shape leads to acceptable closed-loop response when the controller given by (34) is implemented. Clearly the controller must be synthesized from a state-space model of $\mathbf{G}_{W}$ rather than $\mathbf{G}$.

\section{Normalized $\mathcal{H}_{\infty}$ Controller Reduction}

It follows from (34) that the degree of the controller is the same as that of the plant. In our case, the plant's McMillan degree is 76 and this would lead to a correspondingly high-order controller.

In this section, we apply the relative error controller order reduction procedure given in [14]. The main contribution of [14] is to provide a procedure whereby normalized coprime factor controllers can be reduced, whilst giving a priori guaranteed performance bounds. In this reference, it is shown that the observability Gramian of the realization of $\Theta$ in (32), and the controllability Gramian of $\Theta^{-1}$ in (33), may be expressed in terms of $X$ and $Y$. These matrices are the unique positive-definite solutions of (28) and (29), respectively.

Theorem V.1: [14]: Let $P=P^{\prime} \geq 0$ be the controllability Gramian of the realization of $\Theta^{-1}$ in Lemma IV.1 and let $Q=$ $Q^{\prime} \geq 0$ be the observability Gramian of the realization of $\Theta$ in Lemma IV.1 so that

$$
\begin{aligned}
& {\left[Z\left(A-Y C^{\prime} C\right) Z^{-1}\right] P+P\left[Z\left(A-Y C^{\prime} C\right) Z^{-1}\right]^{\prime}} \\
& \quad+Z B B^{\prime} Z^{\prime}+Z Y C^{\prime} C Y Z^{\prime}=0 \\
& \left(A-B B^{\prime} X\right)^{\prime} Q+Q\left(A-B B^{\prime} X\right)+\beta^{-4} X B B^{\prime} X \\
& \quad+\beta^{-4} C^{\prime} C=0 .
\end{aligned}
$$

Then $P=Z Y Z^{\prime}$ and $Q=\beta^{-4} X$.

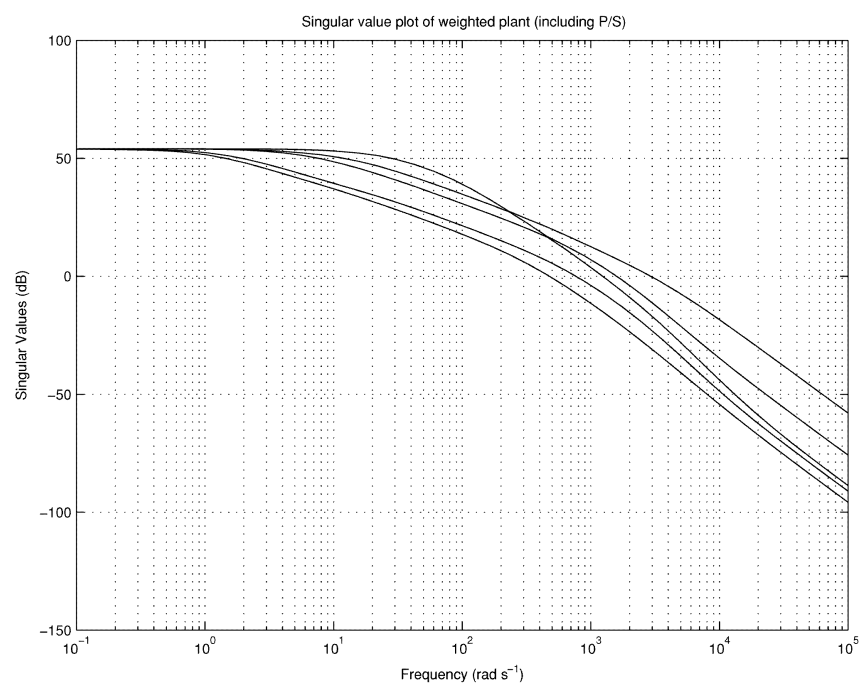

Fig. 7. Response of the weighted plant.

Suppose that the realization of $\Theta$ is inverse-weighted balanced, so that

$$
P=Q=\operatorname{diag}\left(\Sigma_{1}, \Sigma_{2}\right)
$$

where

$$
\begin{aligned}
& \Sigma_{1}=\operatorname{diag}\left(\sigma_{1} I_{s_{1}}, \ldots, \sigma_{r} I_{s_{r}}\right) \\
& \Sigma_{2}=\operatorname{diag}\left(\sigma_{r+1} I_{s_{r+1}}, \ldots, \sigma_{N} I_{s_{N}}\right)
\end{aligned}
$$

with $\sigma_{1}>\cdots \sigma_{N} \geq 0$ and that the realization in (32) is partitioned compatibly with $\Sigma_{1}$ and $\Sigma_{2}$

$$
\boldsymbol{\Theta} \stackrel{s}{=}\left[\begin{array}{cc|c}
A_{11} & A_{12} & B_{1} \\
A_{21} & A_{22} & B_{2} \\
\hline C_{1} & C_{2} & D_{\Theta}
\end{array}\right]
$$

where $D_{\Theta}=\left[\begin{array}{cc}I & 0 \\ 0 & \beta I\end{array}\right]$. Then

$$
\hat{\boldsymbol{\Theta}}=\left[\begin{array}{ll}
\hat{\boldsymbol{\Theta}}_{11} & \hat{\mathbf{\Theta}}_{12} \\
\hat{\mathbf{\Theta}}_{21} & \hat{\mathbf{\Theta}}_{22}
\end{array}\right] \stackrel{s}{=}\left[\begin{array}{l|l}
A_{11} & B_{1} \\
\hline C_{1} & D_{\boldsymbol{\Theta}}
\end{array}\right]
$$

is stable and minimum phase and $\hat{\boldsymbol{\Theta}}=(\mathbf{I}+\boldsymbol{\Delta}) \boldsymbol{\Theta}$, where $\boldsymbol{\Delta} \in$ $\mathcal{R} \mathcal{H}_{\infty}$ satisfies $\|\boldsymbol{\Delta}\|_{\infty} \leq \delta$ where [20]

$$
\delta:=-1+\prod_{i=r+1}^{N}\left(1+2 \sigma_{i} \sqrt{1+\sigma_{i}^{2}}+2 \sigma_{i}^{2}\right)
$$

and

$$
\sigma_{i}^{2}=\frac{\nu_{i}^{2}}{\beta^{4}}\left(1-\frac{\nu_{i}^{2}}{\gamma^{2} \beta^{2}}\right)^{-2} .
$$

The quantities $\nu_{1}^{2}, \ldots, \nu_{N}^{2}$ are the (distinct) eigenvalues of $X Y$.

Since the central controller is obtained from the controller generator in the form of the right coprime factorization (34), it is shown in [14] that the approximation of $\Theta$ induces a relative reduction on the coprime factors of the normalized central controller. Combined with the bound on the relative 
approximation error, this gives guaranteed a priori bounds on closed-loop stability and performance degradation and provides an intimate link between the controller synthesis and controller reduction problems. These results are summarized in the following theorem:

Theorem V.2: [14]: Let $\mathbf{K}_{11}=\boldsymbol{\Theta}_{12} \boldsymbol{\Theta}_{22}^{-1}$ be the central controller defined in (34). Suppose that $T$ is an inverse-weighted balancing transformation for $\Theta$ so that

$$
\begin{aligned}
\Sigma & =T^{-1}\left(Z Y Z^{\prime}\right)\left(T^{-1}\right)^{\prime}=T^{\prime}\left(\beta^{-4} X\right) T \\
& =\operatorname{diag}\left(\sigma_{1} I_{s_{1}}, \ldots, \sigma_{r} I_{s_{r}}, \sigma_{r+1} I_{s_{r+1}}, \ldots, \sigma_{N} I_{s_{N}}\right) \\
& =\operatorname{diag}\left(\Sigma_{1}, \Sigma_{2}\right)
\end{aligned}
$$

with $\sigma_{1}>\cdots>\sigma_{N} \geq 0$. Apply the similarity transformation $T$ to the central controller $\mathbf{K}_{11}$ and partition compatibly with $\Sigma_{1}$ and $\Sigma_{2}$

$$
\begin{aligned}
\mathbf{K}_{11} & \stackrel{s}{=}\left[\begin{array}{l|l}
T^{-1}\left(A-B B^{\prime} X-\beta^{-2} Z Y C^{\prime} C\right) T & T^{-1}\left(Z Y C^{\prime}\right) \\
\hline\left(-\beta^{-2} B^{\prime} X\right) T & 0
\end{array}\right] \\
& \stackrel{s}{=}:\left[\begin{array}{ll|r}
\hat{A}_{11} & \hat{A}_{12} & \hat{B}_{1} \\
\hat{A}_{21} & \hat{A}_{22} & \hat{B}_{2} \\
\hline \hat{C}_{1} & \hat{C}_{2} & 0
\end{array}\right] .
\end{aligned}
$$

Define the reduced order controller

$$
\hat{\mathbf{K}}:=\hat{\boldsymbol{\Theta}}_{12} \hat{\boldsymbol{\Theta}}_{22}^{-1} \stackrel{s}{=}\left[\begin{array}{c|c}
\hat{A}_{11} & \hat{B}_{1} \\
\hline \hat{C}_{1} & 0
\end{array}\right] .
$$

Then $\mathcal{H}\left(\mathbf{G}_{W}, \hat{\mathbf{K}}\right)$ is stable if $\gamma \delta<1$, in which case

$$
\left\|\mathcal{H}\left(\mathbf{G}_{W}, \hat{\mathbf{K}}\right)\right\|_{\infty}<\frac{\gamma}{1-\delta \gamma}
$$

\section{CONTROLLER REDUCTION IMPLEMENTATION}

The final phase of the work is aimed at the implementation and testing of the reduced order controller described in Theorem V.2.

Preliminary testing was conducted on the linear RZIP model. Once the tests were deemed satisfactory, more demanding simulations were performed on the nonlinear PROTEUS model [18].

This program began with the synthesis of a full-order normalized coprime factor controller. The synthesis used the formula (34) and a suboptimal $\gamma=3.02$. The controller realization was then balanced in accordance with Theorem V.2 so that reduced order controllers can be "read off." This sequence of reduced order controllers was combined with $\mathbf{G}_{W}$ in order to generate the plot shown in Fig. 8. This diagram shows, for each reduced order controller, the infinity norm of the closed-loop transfer function as well as the upper bound given in (38). It is clear from this diagram that the closed-loop performance improves very little for controllers of McMillan degree $\geq 16$. The upper bound is clearly conservative and indicates that a controller of degree $\geq 30$ is required. It should also be noted that the reduced

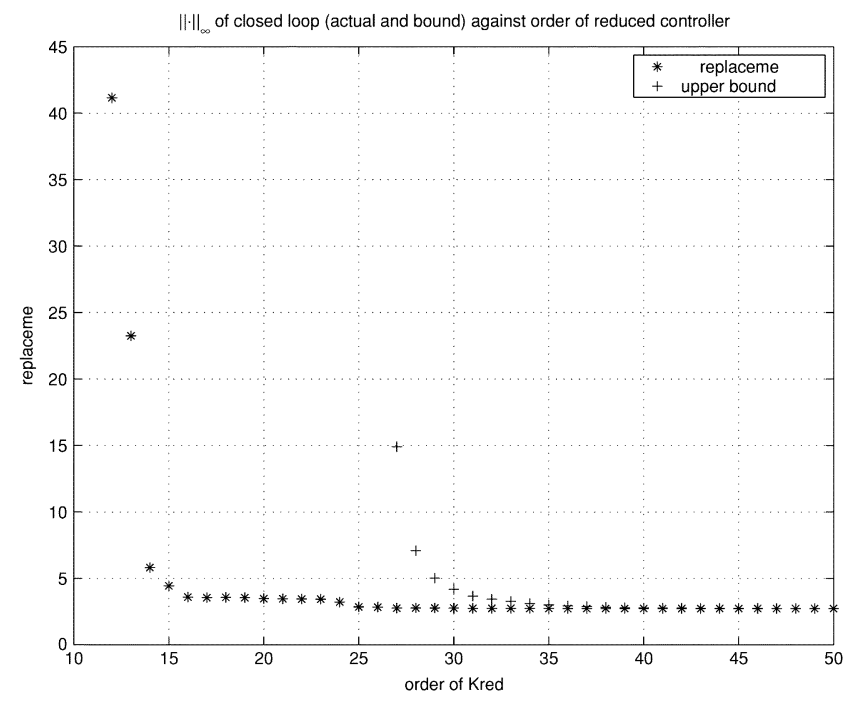

Fig. 8. Plot of $\left\|\mathcal{F}_{l}\left(\mathbf{G}_{W}, \hat{\mathbf{K}}\right)\right\|_{\infty}(*)$ against the order of the reduced controller. Closed loop is unstable for order less than 12 . The a priori bound on $\left\|\mathcal{F}_{l}\left(\mathbf{G}_{W}, \hat{\mathbf{K}}\right)\right\|_{\infty}$ is shown as a (+) where stability is a priori guaranteed.

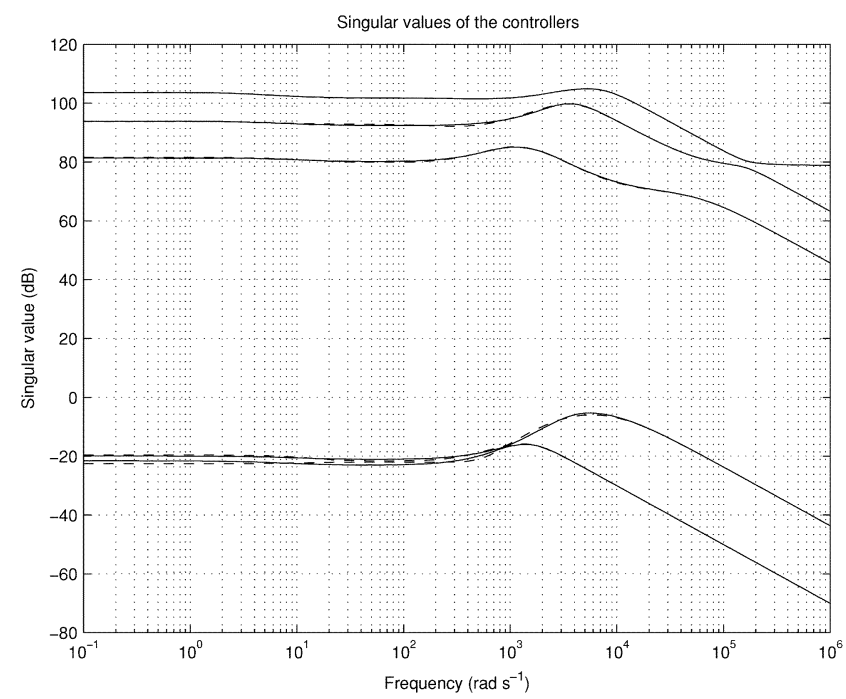

Fig. 9. Singular value plots of the (-) $\gamma=3.02$ controller, (- $)$ reduced $\gamma=$ 3.91 controller (18 states).

order controllers are stabilising for order $\geq 12$, while the bounds in Theorem V.2 indicate that this lowest order for stability is 27.

On the basis of the data in Fig. 8, a controller of order 18 was selected. This represents a significant reduction from the full order of 76 .

Figs. 9 and 10 show the effect of the reduction process on the controller and closed-loop transfer functions, respectively. Fig. 9 shows that the reduction from 76 to 18 states has almost no effect on the controller transfer function. Fig. 10 shows that the closed-loop infinity norm is increased from $\gamma=3.02$ to $\gamma=3.91$.

\section{CONTROLler Simulation Testing}

Even on a relatively small tokamak such as TCV, experimental time and machine failures are potentially expensive. In order to obviate these difficulties, we undertook a program of 


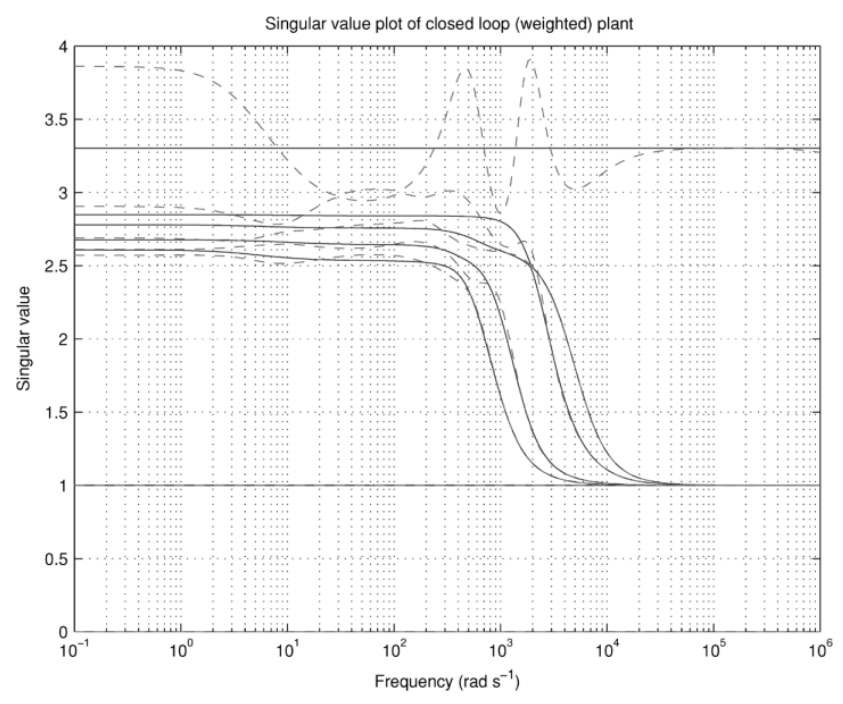

Fig. 10. Singular value plot for the weighted closed-loop plant with $(-) \gamma=$ 3.02 and $(--)$ reduced $\gamma=3.91$ controller.

controller testing at a simulation level prior to any hardware implementation. The two steps involved simulation testing on RZIP, with a second program of tests on PROTEUS. The outcome of the simulation tests will now be presented.

\section{A. Testing on RZIP in SIMULINK}

Since RZIP models are linear, the only source of nonlinearity in these simulations will be power supply saturation. It is convenient to use the SIMULINK environment to introduce these effects. A full list of the power supply limits is given in the table below [15].

\begin{tabular}{l|l|l}
\hline PF coil P/S & $\begin{array}{l}\text { Saturation } \\
\text { voltage }(+/-)\end{array}$ & $\begin{array}{l}\text { Saturation } \\
\text { current }(+/-)\end{array}$ \\
\hline OH1, OH2 & $1399 \mathrm{~V}$ & $31 \mathrm{kA}$ \\
E1-E8 & $648 \mathrm{~V}$ & $7.7 \mathrm{kA}$ \\
F1-F8 & $1250 \mathrm{~V}$ & $7.7 \mathrm{kA}$ \\
G & $566 \mathrm{~V}$ & $500 \mathrm{~A}($ eontinuous) \\
& & $2000 \mathrm{~A}(2 \mathrm{~s} /$ min $)$ \\
\hline
\end{tabular}

For the purposes of this study, we designed a controller that combines good robust stability margins, speed of response, dynamic tracking characteristics, and closed-loop decoupling. In order to test for these various characteristics, we drove the closed-loop system with a vector valued reference signal that comprised pulse functions that were staggered in time. The pulse amplitudes were chosen to represent "reasonable" but demanding excursions given the machine dimensions. ${ }^{4}$ The temporal separation between the pulses allowed us to examine the interloop crosscoupling. The performance of the 18-state reduced order controller is shown in Fig. 11. It is clear from the responses that the controller possesses all the desired properties; the pulse inputs lie underneath the responses and are barely visible on the plot. This is testimony to the controller's excellent tracking properties. It is also clear that the only crosscoupling appears at the pulse edges.

\footnotetext{
${ }^{4}$ This includes both the machine geometry and the electromagnetic specifications.
}
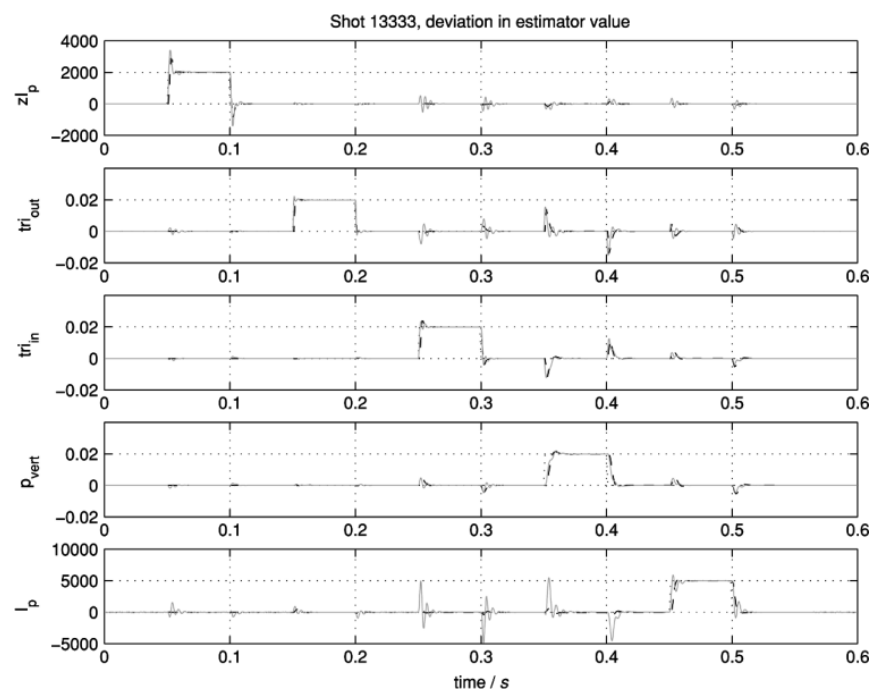

Fig. 11. Results of closed-loop simulation showing (:) reference and (-) RZIP simulated response.

Although saturation limits were included in the simulation, these played very little role in the particular responses shown here. This is because the TCV power supplies are deliberately over-designed.

\section{B. Testing on the PROTEUS Nonlinear Tokamak Simulation Code}

PROTEUS is a nonlinear tokamak simulation code that solves the Grad-Shafranov equation using an iterative finite element method. As such it can be used to predict the tokamak plasma's evolution forward in time, under the assumption of a fixed plasma current. In order to initialize these simulations, PROTEUS requires data about the initial equilibrium, specifically the coil currents and some plasma profile parameters.

Although PROTEUS can model temporal changes in the plasma shape, it does not take account of transport and resistivity phenomena that are known to occur. Since the original code does not have a plasma circuit equation, one had to be introduced, although this is not strictly self-consistent with the initial assumptions. The controller acts to change the plasma current so this dynamic response must be represented.

In order to complete the simulation work presented here, we had to introduce a facility for modeling discrete-time controllers and estimator matrices that generate the controlled plant outputs.

The issue of delays introduced by discretization of the feedback controllers has been addressed in [12], in which a highorder $\mathcal{H}_{\infty}$ controller was tested on the TCV tokamak. The continuous-time controller used here was discretized with a time step of $10^{-4} \mathrm{~s}$, prior to testing on PROTEUS. TCV diagnostics are capable of this sampling frequency.

The PROTEUS simulations represent a form of robustness test, because the code is significantly different to the RZIP model used in the controller synthesis. The successful operation of the PROTEUS closed-loop therefore lends confidence to the idea that the controller will work successfully on the hardware system. 

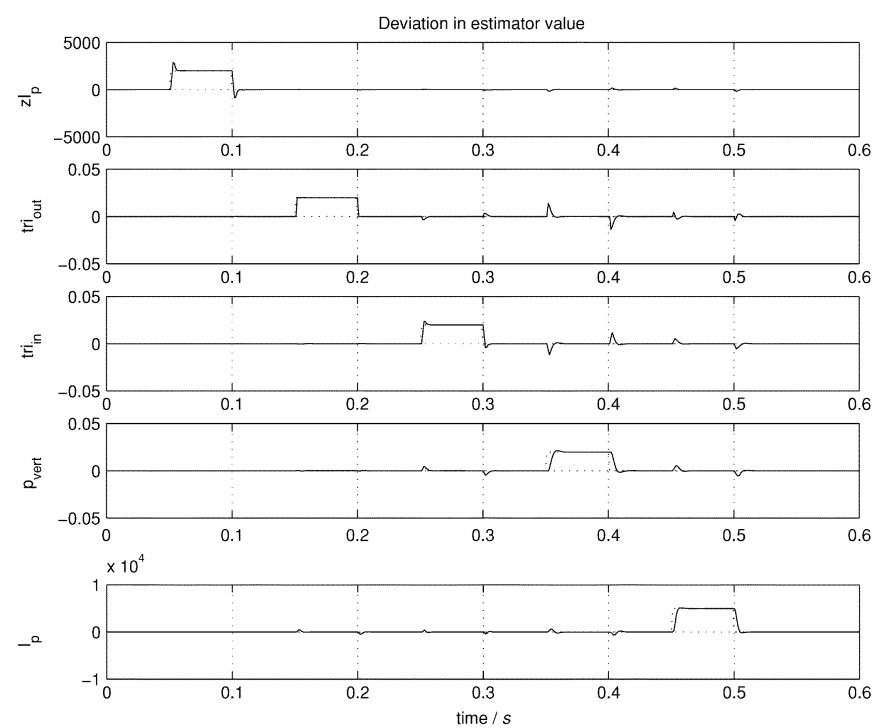

Fig. 12. Results of closed-loop simulations, showing (-) the PROTEUS simulated response, (:) the reference and (--) the RZIP simulated response.
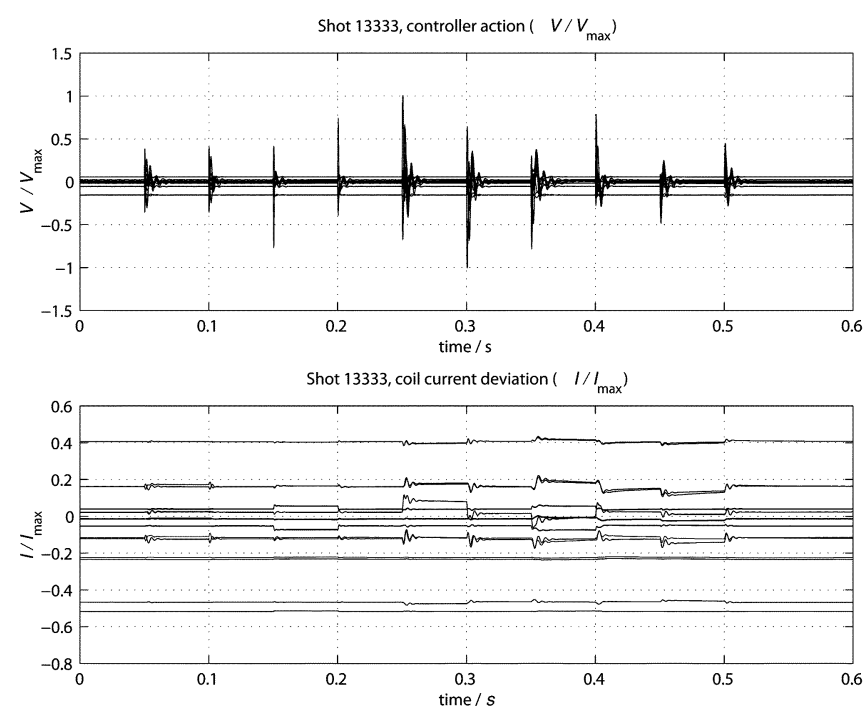

Fig. 13. Closed-loop simulation coil voltage and current.

Fig. 12 shows the results of the PROTEUS simulation with a 0.1 -ms time step. To aid comparison, the RZIP results are shown on the same diagram. The input and nominal equilibrium conditions were chosen to match those associated with the RZIP results. It can be seen from Fig. 12 that there is a high level of agreement between the PROTEUS and the RZIP predictions. With that said, one can see a number of lightly damped resonances in the PROTEUS plasma current that are absent in the RZIP equivalent. This is considered likely to represent the inconsistencies in the PROTEUS modeling of the plasma circuit equation.

The practical import of this close agreement is that RZIP simulations that require a few minutes to compute can be used instead of PROTEUS predictions that require days on a like-forlike basis.
Fig. 13 shows the coil voltage and current as a fraction of the saturation limits. This diagram shows that the power supplies do not saturate except very briefly at 0.25 and $0.3 \mathrm{~s}$ where the inner triangularity is stimulated. This does not lead to a loss of control in the simulation. Since the slight saturation occurs only when the inner triangularity is stimulated hard, and this parameter is unlikely to be pulsed in normal TCV operation, the saturation is unlikely to lead to a loss of plasma control on TCV.

\section{DISCUSSION}

We have presented a new modeling and control system design paradigm for tokamak fusion reactors. From the initial modeling assumptions, the methodology takes us through the modeling process to low-order controllers with good performance and robustness characteristics.

Starting with clearly stated assumptions, a nonlinear model together with its linearized counterpart are derived using known techniques from Hamiltonian mechanics. This approach to the modeling problem clarifies many of the relationships between the assumptions and the model properties. For example, it becomes clear that current ramping is necessary to maintain the plasma current when the plasma resistance is nonzero. Since future machines will operate on longer time-scales, plasma resistance effects will become more important. The model presented here incorporates plasma resistance thereby satisfying this need. The effect of removing plasma resistance from the model is also clarified. We show that this leads to the introduction of uncontrollable modes at the origin. This is examined further in the Appendix.

The second part of the paper demonstrates the effectiveness of a novel model reduction methodology for normalized coprime factor controller reduction. The known procedures of normalized coprime factor controller design produce control systems with order equal to that of the loop-shaped plant [20], [24]. Since we have used nondynamic weights, the controller order is 76 according to this established wisdom. We have demonstrated that it is possible to reduce the controller order to 18 using the techniques described in [14] without a significant performance degradation. The a priori bounds on closed-loop performance given in [14] are found to be quite conservative, giving a stabilising reduced order controller of minimum order 27. It was also demonstrated that this performance was successfully transferred to the nonlinear tokamak simulation code PROTEUS. In this context, this successful transfer is indicative of the inherent robustness properties of the controller. This is because PROTEUS is based on modeling assumptions quite different to those used in the model presented here, on which the controller synthesis was based. Given the successful transfer to PROTEUS, we are confident that good performance will be achieved on the TCV hardware using this methodology.

\section{APPENDIX \\ EXAMPLE OF A COUPLED LR CIRCUIT}

The purpose of this Appendix is to illustrate, using the simple inductance-resistance (LR) circuit in Fig. 14, the mechanism behind the creation of uncontrollable modes in tokamak models 


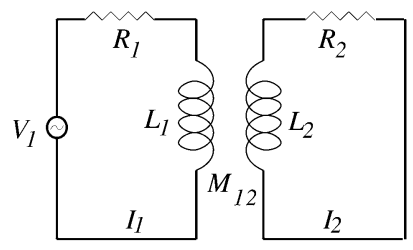

Fig. 14. Coupled LR circuit.

containing idealized superconducting plasmas. ${ }^{5}$ To the author's knowledge this problem has not been discussed previously.

In this diagram the secondary circuit represents the plasma, the primary circuit represents the tokamak coils and the mutual inductance the magnetic coupling between them.

It follows from simple circuit theory that

$$
\left[\begin{array}{l}
1 \\
0
\end{array}\right] V_{1}=\left[\begin{array}{cc}
L_{1} & M_{12} \\
M_{12} & L_{2}
\end{array}\right]\left[\begin{array}{c}
\dot{I}_{1} \\
\dot{I}_{2}
\end{array}\right]+\left[\begin{array}{cc}
R_{1} & 0 \\
0 & R_{2}
\end{array}\right]\left[\begin{array}{l}
I_{1} \\
I_{2}
\end{array}\right]
$$

which can be put into state-space form

$$
\begin{aligned}
{\left[\begin{array}{l}
1 \\
0
\end{array}\right] u } & =\mathcal{M} \dot{x}+\mathcal{R} x \\
& \Rightarrow \dot{x}=-\mathcal{M}^{-1} \mathcal{R} x+\mathcal{M}^{-1}\left[\begin{array}{l}
1 \\
0
\end{array}\right] u
\end{aligned}
$$

with

and

$$
A=-\mathcal{M}^{-1} \mathcal{R}
$$

$$
B=\left[\begin{array}{l}
\alpha \\
\gamma
\end{array}\right]
$$

where

$$
\mathcal{M}^{-1}=\left[\begin{array}{ll}
\alpha & \beta \\
\gamma & \delta
\end{array}\right]
$$

Observe that $\mathcal{M}$ is necessarily invertible because $L_{1}>M_{12}$ and $L_{2}>M_{12}$.

Hence

$$
A=-\left[\begin{array}{cc}
\alpha R_{1} & \beta R_{2} \\
\gamma R_{1} & \delta R_{2}
\end{array}\right]
$$

It follows from standard passivity arguments that $\operatorname{Re} \lambda_{i}(A)<$ 0 for $R_{1}, R_{2}>0$.

The controllability matrix is

$$
\left[\begin{array}{c|c|c}
s I-A & \mid & B
\end{array}\right]=\left[\begin{array}{cc|c}
s+\alpha R_{1} & \beta R_{2} & \alpha \\
\gamma R_{1} & s+\delta R_{2} & \gamma
\end{array}\right] .
$$

It is therefore the case that $(A, B)$ is controllable if $R_{2}>0$. On the other hand, if $R_{2}=0$, the matrix in (41) will have unit rank at $s=0$, indicating the presence of an uncontrollable mode there.

\footnotetext{
${ }^{5}$ The results are also relevant to tokamaks with superconducting coils such as ITER. However, in that case we would not simply be able to ignore the resulting uncontrollable modes.
}

In reality, we will never face the $R_{2}=0$ situation and so the associated uncontrollable mode may be ignored.

In the case that $R_{2}=0$, it follows that

$$
I_{2}=-\frac{M_{12}}{L_{2}} I_{1}+C
$$

demonstrating that under these conditions the two loop currents are dependent.

We can also see that

$$
\dot{I}_{1}=-\frac{I_{2} R_{2}}{M_{12}}
$$

in the case that $I_{2}$ is constant, which illustrates the need to ramp $I_{1}$ in order that $I_{2}$ be maintained at a fixed value.

\section{ACKNOWLEDGMENT}

The authors would like to thank J. Wainwright for the generosity with which he gave his time to help set up PROTEUS, and for lively discussions and ideas about the modeling.

\section{REFERENCES}

[1] V. Mukhovatov and V. Shafranov, "Plasma equilibrium in a tokamak," Nucl. Fusion, vol. 11, p. 605, 1971.

[2] J. Wesson, Tokamaks. Oxford, U.K.: Oxford Univ. Press, 1997.

[3] R. Albanese and F. Villone, "The linearized CREATE-L model for the control of current, position, and shape in tokamaks," Nucl. Fusion, vol. 38 , no. 5, pp. 723-738, 1998.

[4] D. Humphreys and I. Hutchinson, "Axisymmetric magnetic control design in tokamaks using perturbed equilibrium plasma response modeling," Fusion Technol., vol. 23, p. 167, 1993.

[5] D. Humphreys, J. Leuer, and M. Walker, "Minimal plasma response models for design of tokamak equilibrium controllers with high dynamic accuracy," in Proc. 41st Annu. Meeting of Division of Plasma Physics, Seattle, WA, Nov. 15-19, 1999, p. 175.

[6] A. Coutlis, I. Bandyopapadhyay, J. Lister, P. Vyas, R. Albanese, D. Limebeer, F. Villone, and J. Wainwright, "Measurement of the open loop plasma equilibrium response in TCV," Nucl. Fusion, vol. 39, no. 5, pp. 663-683, 1999.

[7] F. Hofmann, J.-M. Moret, and D. Ward, "Stability analysis of the vertical position control loop in TCV using rigid and deformable plasma models," Nucl. Fusion, vol. 38, no. 12, pp. 1767-1778, 1998.

[8] A. Portone, R. Albanese, Y. Gribov, M. Huguet, D. Humphreys, C. Kessel, P.-L. Mondino, L. D. Pearlstein, and J. Wesley, "Dynamic control of plasma position and shape in ITER," Fusion Technol., vol. 32, pp. 374-389, 1997.

[9] J. Lister, A. Sharma, D. Limebeer, Y. Nakamura, J. Wainwright, and R. Yoshino, "Plasma equilibrium response modeling and validation on JT-60U," Nucl. Fusion, vol. 42, pp. 708-724, 2002.

[10] E. Lazarus, J. Lister, and G. Neilson, "Control of the vertical instability in tokamaks," Nucl. Fusion, vol. 30, no. 1, p. 111, 1990.

[11] P. Vyas, "Plasma vertical position control on COMPASS-D," Ph.D. dissertation, Dept. Eng. Sci., Oxford Univ., Oxford, U.K., 1996.

[12] M. Ariola, G. Ambrosino, J. Lister, A. Pironti, F. Villone, and P. Vyas, "A modern plasma controller tested on the TCV tokamak," Fusion Technol., vol. 36, pp. 126-138, 1999.

[13] M. Walker, D. Humphreys, and J. Ferron, "Control of plasma poloidal shape and position in the DIII-D tokamak," in Proc. 36th Conf. Decision and Control, San Diego, CA, Dec. 1997, p. 3703.

[14] H. El-Zobaidi, I. Jaimoukha, and D. Limebeer, "Normalized $\mathcal{H}_{\infty}$ controller reduction with a priori bounds," IEEE Trans. Autom. Control, vol. 46, no. 9, pp. 1477-1483, Sep. 2001.

[15] J. Lister, F. Hofmann, J.-M. Moret, F. Bühlmann, M. Dutch, D. Fasel, A. Favre, P.-F. Isoz, B. Marletaz, P. Marmillod, Y. Martin, A. Perez, and D. Ward, "The control of tokamak configuration variable plasmas," Fusion Technol., vol. 32, pp. 321-373, 1997. 
[16] H. Goldstein, Classical Mechanics, 2nd ed. Reading, MA: AddisonWesley, 1980.

[17] J. Wainwright, D. Copsey, D. Limebeer, M. Haines, and A. Portone, "Extensions to single filament modeling of a tokamak plasma," Nucl. Fusion, vol. 37, no. 12, p. 1697, 1997.

[18] R. Albanese, J. Blum, and O. de Barbieri, "Numerical Studies of the Next European Torus via the Proteus Code," in Proc. 12th Conf. Numerical Simulations of Plasmas, San Francisco, CA, 1987.

[19] M. Safonov and R. Chiang, "A Schur method for balanced-truncation model-reduction," IEEE Trans. Autom. Control, vol. 34, no. 7, pp. 729-733, Jul. 1989.

[20] M. Green and D. Limebeer, Linear Robust Control. Englewood Cliffs, NJ: Prentice-Hall, 1995.

[21] K. Glover and D. McFarlane, "Robust stabilization of normalized coprime factor plant descriptions with $\mathcal{H}_{\infty}$-bounded uncertainty," IEEE Trans. Autom. Control, vol. 34, no. 8, pp. 821-830, Aug. 1989.

[22] A. Portone, "Modeling and Control of tokamak plasmas in fusion devices," Ph.D. dissertation, Dept. Elect. Eng., Univ. London, London, U.K., 1994

[23] K. Zhou, "Frequency-weighted $\mathcal{L}_{\infty}$ norm and optimal Hankel norm model reduction," IEEE Trans. Autom. Control, vol. 40, no. 10, pp. $1687-1699$, Oct. 1995.

[24] D. McFarlane and K. Glover, "A loop-shaping design procedure using $\mathcal{H}_{\infty}$ synthesis," IEEE Trans. Autom. Control, vol. 37, no. 6, pp. 759-769, Jun. 1992.

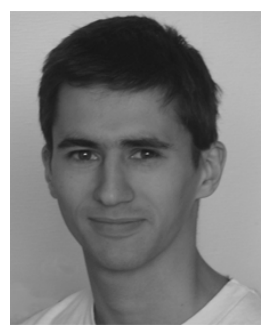

Atul S. Sharma received the M.Sc. degree in physics from University College, London, U.K., in 1998 and the Ph.D. degree in tokamak modeling and control from Imperial College, London, U.K., in 2002.

$\mathrm{He}$ is currently a Research Associate at Imperial College. His interests include control of flapless air vehicles.

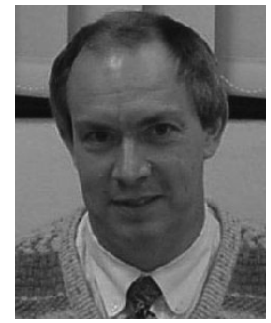

David J. N. Limebeer (M'82-SM'86-F'92) received the B.Sc. degree in electrical from the University of Witwatersrand, Johannesburg, South Africa, in 1974, the M.Sc. degree in electrical engineering and the Ph.D. degree from the University of Natal, Durban, South Africa, in 1977 and 1980, respectively, and the D.Sc. degree from the University of London, London, U.K., in 1992.

He became a Reader at Imperial College, London, U.K., in 1989, and was promoted to Professor in 1993. He was Head of the Control Group between 1995 and 1999 and is currently the Head of the Department of Electrical and Electronic Engineering. He has published over 100 papers in peer reviewed journals and a textbook on robust control theory. He is a past Editor of Automatica and a past Associate Editor of Systems and Control Letters and the International Journal of Robust and Nonlinear Control.

Dr. Limebeer is a Fellow of the Institution of Electrical Engineers (IEE), the Royal Academy of Engineering, and the City and Guilds Institute.

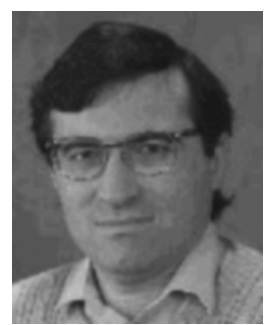

Imad M. Jaimoukha received the B.Sc. degree in electrical engineering from the University of Southampton, U.K., in 1983, the M.Sc. degree in control systems in 1986, and the Ph.D. degree from Imperial College, London, U.K., in 1990.

He was a Research Assistant in the Department of Electrical and Electronic Engineering, Imperial College, London, U.K., before being appointed to his current position as Lecturer. He has published articles in the areas of robust control design, model and controller reduction, and the control of power

systems.

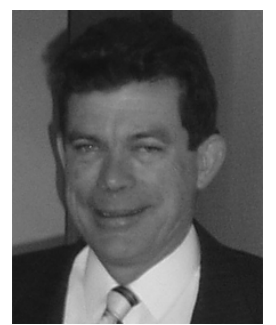

Jonathan B. Lister received the B.A. degree in physics from Oxford University, Oxford, U.K., in 1971 and the Ph.D. degree in high-energy physics from the University of London, U.K., in 1974.

$\mathrm{He}$ is currently a Senior Research Physicist at the Centre de Recherches en Physique des Plasmas, Ecole Polytechnique Federale de Lausanne, Lausanne, Switzerland. His current research interests include advanced plasma control and full nonlinear tokamak simulations, especially as they relate to the control of future large tokamaks. 